\title{
Research Article \\ Decoupled Estimation of 2D DOA for Coherently Distributed Sources Using 3D Matrix Pencil Method
}

\author{
Zhang Gaoyi and Tang Bin \\ School of Electronic Engineering, University of Electronic Science and Technology of China, Chengdu 610054, China
}

Correspondence should be addressed to Zhang Gaoyi, gaoyzhang@163.com

Received 31 January 2008; Revised 17 May 2008; Accepted 13 July 2008

Recommended by S. Gannot

A new 2D DOA estimation method for coherently distributed (CD) source is proposed. CD sources model is constructed by using Taylor approximation to the generalized steering vector (GSV), whereas the angular and angular spread are separated from signal pattern. The angular information is in the phase part of the GSV, and the angular spread information is in the module part of the GSV, thus enabling to decouple the estimation of 2D DOA from that of the angular spread. The array received data is used to construct three-dimensional (3D) enhanced data matrix. The 2D DOA for coherently distributed sources could be estimated from the enhanced matrix by using 3D matrix pencil method. Computer simulation validated the efficiency of the algorithm.

Copyright (c) 2008 Z. Gaoyi and T. Bin. This is an open access article distributed under the Creative Commons Attribution License, which permits unrestricted use, distribution, and reproduction in any medium, provided the original work is properly cited.

\section{INTRODUCTION}

In many applications, such as wireless communications, radar, and sonar, the effect of angular spread can no longer be ignored. A distributed source model will be more appropriate $[1,2]$. Distributed source is classified as coherently distributed (CD) source and incoherently distributed (ICD) source in literature [2], where angular signal density of the sources is used to form the distributed model. When the received signal components from a source at different angles are delayed and scaled replicas of the same signal, the source is called coherently distributed. When the signal rays arriving from different directions are uncorrelated, the source is called incoherently distributed. In $\mathrm{CD}$ source case, the rank of the noise-free covariance matrix is equal to the number of sources. Some classical estimation methods [2-6] were generalized from the case of point sources to the case of CD sources. DSPE [2, 6] is generalized from MUSIC for the distributed sources parameter estimation. ESPRIT is extended for distributed sources parameter estimation by using two closely-spaced ULAs [3]. The above algorithms have mainly been developed for azimuth-only estimation and angular spread. Based on two closely-spaced UCAs, the sequential one-dimensional searching (SOS) method [4] which is the combination of ESPRIT and alternate minimization in $2 \mathrm{D}$ problem is developed in [4], where the nominal azimuth DOA and elevation DOA of coherently distributed sources can be obtained by one-dimensional search. Based on specially designed array geometry, VESPA is used for the estimation of 2D DOA and angular spread for coherently distributed source [5].

In this paper, the Taylor approximation is used to separate the angular information from angular spread information. The angular information can be got from the phase part of the received signal, which can be got from the poles extracted by matrix pencil (MP) method. So, MP method can be used to decouple the estimation of 2D DOA from that of the angular spread for coherently distributed source. The MP method is used for the estimation of twodimensional frequencies in $[7,8]$ and then extended for the 2D DOA estimation for point source in $[9,10]$. We extend it for the 2D DOA estimation of coherently distributed sources without any search. The array received data is used to construct $3 \mathrm{D}$ enhanced data matrix. The signal's $2 \mathrm{D}$ DOA information is extended into $3 \mathrm{D}$ poles along three planes and is expressed by an enhanced matrix. After the three poles are estimated from the phase part of the signal, the 2D DOA for coherently distributed sources could be estimated. 


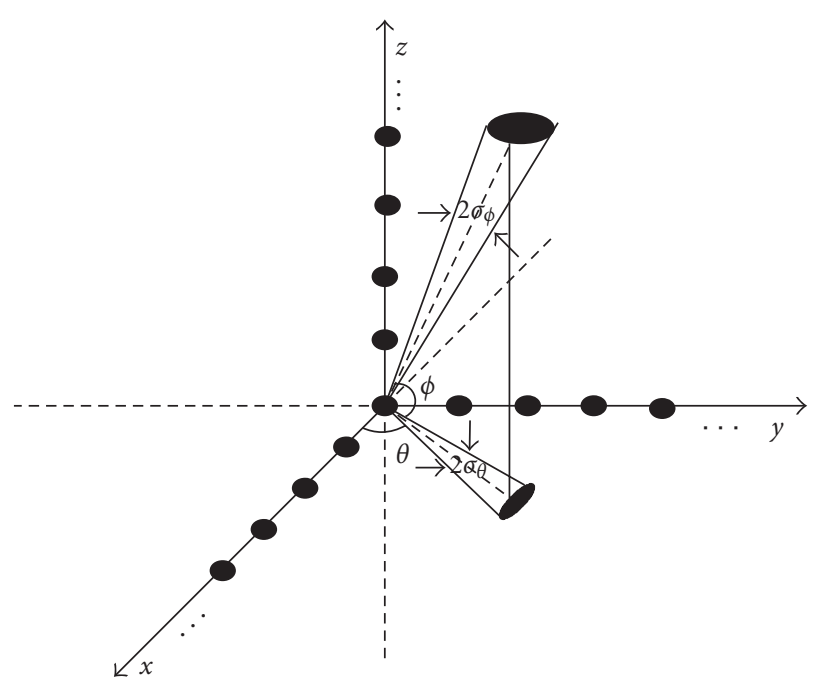

FIgURE 1: Array geometry.

\section{SIGNAL MODEL}

Assume that stationary signals impinge on an array of $K$ sensors from $I$ narrowband far-field sources. The output of the sensors of the array is given by

$$
\mathbf{v}(t)=\mathbf{B s}(t)+\mathbf{n}(t)
$$

where $\mathbf{B}=\left[\mathbf{b}_{1}, \mathbf{b}_{2}, \ldots, \mathbf{b}_{I}\right], \mathbf{s}(t)=\left[s_{1}(t), s_{2}(t), \ldots, s_{I}(t)\right]^{T}$, and $\mathbf{n}(t)$, respectively, are the $K \times I$ generalized steering matrix formed between the sources and the antenna elements at the array, the $I \times 1$ signal vector transmitted by the source, and the $K \times 1$ additive noise vector. $\mathbf{b}_{i}$ is the $K \times 1 \mathrm{GSV}$ of the $i$ th CD source, which is defined as

$$
\mathbf{b}_{i}=\iint \mathbf{a}(\theta, \phi) \rho\left(\theta, \phi ; \mu_{i}\right) d \theta d \phi
$$

where $\mathbf{a}(\theta, \phi)$ is the steering vector for a point source at $2 \mathrm{D}$ $\operatorname{DOA}(\theta, \phi), \mu_{i}=\left(\theta_{i}, \sigma_{\theta_{i}}, \phi_{i}, \sigma_{\phi_{i}}\right)$ is a vector whose elements, respectively, are the azimuth DOA, the angular spread of the azimuth DOA, the elevation DOA, and the angular spread of the elevation DOA of the $i$ th CD source. $\rho\left(\theta, \phi ; \mu_{i}\right)$ is the deterministic angular weighting function of the $i$ th $\mathrm{CD}$ source.

Similar to [4], when the angular spread is small, for a single CD source narrowband centered in $\lambda$, we can use the Taylor approximation, the elements of GSV can be approximately decomposed as

$$
b_{k}(\mu)=\left[\mathbf{b}\left(\theta, \sigma_{\theta}, \phi, \sigma_{\phi}\right)\right]_{k} \approx[\mathbf{a}(\theta, \phi)]_{k}\left[\mathbf{g}\left(\theta, \sigma_{\theta}, \phi, \sigma_{\phi}\right)\right]_{k},
$$

where $[\mathbf{a}(\theta, \phi)]_{k}$ is the phase part of the GSV, and $\left[\mathbf{g}\left(\theta, \sigma_{\theta}, \phi, \sigma_{\phi}\right)\right]_{k}$ is the module part of the GSV.

Consider a three-dimensional array in space as illustrated in Figure 1 with the axes oriented along the Cartesian coordinates. The distances between the elements are $\Delta x, \Delta y$, and $\Delta z$, which are always half of the wavelength.
The number of sensors are $A, B$, and $C$ satisfying $A+B+$ $C=K$. Therefore, we have

$$
\begin{aligned}
& {[\mathbf{a}(\theta, \phi)]_{(a, b, c)}} \\
& \quad=e^{j((2 \pi / \lambda) \Delta x \cos \theta \cos \phi a+(2 \pi / \lambda) \Delta y \sin \theta \cos \phi b+(2 \pi / \lambda) \Delta z \sin \phi c)},
\end{aligned}
$$

where $a=1, \ldots, A ; b=1, \ldots, B ; c=1, \ldots, C$; and

$$
\begin{aligned}
{\left[\mathbf{g}\left(\theta, \sigma_{\theta}, \phi, \sigma_{\phi}\right)\right]_{(a, b, c) \text { Gaussian }} } & \\
= & e^{-2 \pi^{2} \sigma_{\theta}^{2}(-\Delta x \sin \theta \cos \phi a+\Delta y \cos \theta \cos \phi b)^{2} / \lambda^{2}} \\
& \times e^{-2 \pi^{2} \sigma_{\phi}^{2}(-\Delta x \cos \theta \sin \phi a-\Delta y \sin \theta \sin \phi b+\Delta z \cos \phi c)^{2} / \lambda^{2}}
\end{aligned}
$$

for Gaussian shaped coherently distributed (GCD) source,

$$
\begin{gathered}
{\left[\mathbf{g}\left(\theta, \sigma_{\theta}, \phi, \sigma_{\phi}\right)\right]_{(a, b, c) \text { Laplacian }}} \\
=1 /\left(\left(1+2\left(\pi \sigma_{\theta}(-\Delta x \sin \theta \cos \phi a+\Delta y \cos \theta \cos \phi b) / \lambda\right)^{2}\right)\right. \\
\times\left(1+2\left(\pi \sigma_{\phi}(-\Delta x \cos \theta \sin \phi a-\Delta y \sin \theta \sin \phi b\right.\right. \\
\left.\left.+\Delta z \cos \phi c) / \lambda)^{2}\right)\right)
\end{gathered}
$$

for Laplacian shaped coherently distributed (LCD) source.

It is noted that when the angular spread of coherently distributed source is small, for Gaussian shaped and Laplacian shaped distributed source, the angular information and angular spread information could be separated from the signal pattern. From (3), (4), (5), and (6), we know that the angular spread only affect the module of the received signal because MP algorithm extracts the poles from the phase of signal, the MP algorithm might be used for the estimation of 2D DOA similar as [9] for point source. For differently shaped coherently distributed source, the 2D DOA can be decoupled from the angular spreads by using MP algorithm. So, the MP algorithm can obtain the 2D DOA of coherently distributed sources without the prior information of the shape of the angular weighting function. Obviously, when $\sigma_{\theta}=0$ and $\sigma_{\phi}=0$, the above model is a point model. When the angular spread increases, the module of the coherently distributed sources decreases.

\section{MATRIX PENCIL METHOD}

Assume the $i$ th coherently distributed source signals are narrowband centered in $\lambda_{i}$, consider a $3 \mathrm{D}$ data cube as

$$
\begin{aligned}
& v(a ; b ; c) \\
& =\sum_{i=1}^{I} e^{j\left(\left(2 \pi / \lambda_{i}\right) \Delta x \cos \theta_{i} \cos \phi_{i} a+\left(2 \pi / \lambda_{i}\right) \Delta y \sin \theta_{i} \cos \phi_{i} b+\left(2 \pi / \lambda_{i}\right) \Delta z \sin \phi_{i} c\right)} \alpha_{i} \\
& \quad+w(a, b, c)
\end{aligned}
$$

where $w(a, b, c)$ denotes the noise, and

$$
\alpha_{i}=g(i)_{(a, b, c)} s_{i}(t)=\left[\mathbf{g}\left(\theta_{i}, \sigma_{\theta_{i}}, \phi_{i}, \sigma_{\phi_{i}}\right)\right]_{(a, b, c)} s_{i}(t),
$$


where $s_{i}(t)=M_{i} e^{j \gamma_{i}}$ is the signal with amplitude of $M_{i}$ along with the phase $\gamma_{i}$.

Define $3 \mathrm{D}$ poles $x_{i}, y_{i}$, and $z_{i}$ as follows:

$$
\begin{aligned}
& x_{i}=\exp \left(j \frac{2 \pi}{\lambda_{i}} \Delta x \cos \theta_{i} \cos \phi_{i}\right), \\
& y_{i}=\exp \left(j \frac{2 \pi}{\lambda_{i}} \Delta y \sin \theta_{i} \cos \phi_{i}\right), \\
& z_{i}=\exp \left(j \frac{2 \pi}{\lambda_{i}} \Delta z \sin \phi_{i}\right) .
\end{aligned}
$$

After the poles are found, the elevation and the azimuth angle are obtained for each source as follows:

$$
\begin{gathered}
G_{i}=\frac{\operatorname{angle}\left(x_{i}\right)}{2 \pi \Delta x}, \quad E_{i}=\frac{\operatorname{angle}\left(y_{i}\right)}{2 \pi \Delta y}, \quad F_{i}=\frac{\operatorname{angle}\left(z_{i}\right)}{2 \pi \Delta z}, \\
\theta_{i}=\arctan \left(\frac{E_{i}}{G_{i}}\right) \\
\phi_{i}=\arctan \left(\frac{F_{i}}{\sqrt{G_{i}^{2}+E_{i}^{2}}}\right) .
\end{gathered}
$$

The 3D data matrix can be enhanced by using the partition and stacking process. The column vectors along $x$ direction are enhanced by a pencil parameter $L$ and they are stacked to get $\mathbf{D}_{y, z}$ as follows:

$$
\begin{aligned}
& \mathbf{D}_{y, z} \\
& =\left[\begin{array}{cccc}
v(0 ; y ; z) & v(1 ; y ; z) & \cdots & v(A-L ; y ; z) \\
v(1 ; y ; z) & v(2 ; y ; z) & \cdots & v(A-L+1 ; y ; z) \\
\vdots & \vdots & \ddots & \vdots \\
v(L-1 ; y ; z) & v(L ; y ; z) & \cdots & v(A-1 ; y ; z)
\end{array}\right]_{L(A-L+1)}
\end{aligned}
$$

The matrix $\mathbf{D}_{y, z}$ is enhanced along $y$-direction with the pencil parameter $M$ as follows:

$$
\mathbf{D}_{z}=\left[\begin{array}{cccc}
\mathbf{D}_{0, z} & \mathbf{D}_{1, z} & \cdots & \mathbf{D}_{B-M, z} \\
\mathbf{D}_{1, z} & \mathbf{D}_{2, z} & \cdots & \mathbf{D}_{B-M+1, z} \\
\vdots & \vdots & \ddots & \vdots \\
\mathbf{D}_{M-1, z} & \mathbf{D}_{M, z} & \cdots & \mathbf{D}_{B-1, z}
\end{array}\right]_{L M(A-L+1)(B-M+1)}
$$

The matrix $\mathbf{D}_{z}$ is enhanced along $z$-direction with the pencil parameter $N$ as follows:

$$
\mathbf{D}_{e}=\left[\begin{array}{cccc}
\mathbf{D}_{0} & \mathbf{D}_{1} & \cdots & \mathbf{D}_{C-N} \\
\mathbf{D}_{1} & \mathbf{D}_{2} & \cdots & \mathbf{D}_{C-N+1} \\
\vdots & \vdots & \ddots & \vdots \\
\mathbf{D}_{N-1} & \mathbf{D}_{N} & \cdots & \mathbf{D}_{C-1}
\end{array}\right]_{L M N(A-L+1)(B-M+1)(C-N+1)}
$$

The enhanced data matrix $\mathbf{D}_{e}$ is used to obtain the $3 \mathrm{D}$ poles [9]. The singular value decomposition of matrix $\mathbf{D}_{e}$ has the form

$$
\mathbf{D}_{e}=\mathbf{U}_{S} \boldsymbol{\Lambda}_{S} \mathbf{V}_{S}^{H}+\mathbf{U}_{n} \boldsymbol{\Lambda}_{n} \mathbf{V}_{n}^{H}
$$

where $H$ denotes the conjugate transpose, the subindexes $S$ and $n$ stand for the signal and noise components, respectively.

As discussed in [9], the pencil parameter must be chosen to satisfy two relationships with the number of signal as follows:

$$
\begin{gathered}
L M N \geq I, \\
(A-L+1)(B-M+1)(C-N+1) \geq I .
\end{gathered}
$$

In CD source case, the rank of the noise-free covariance matrix is equal to the number of sources. The algorithm can be summarized as follows.

Step 1. Form the $L M N \times(A-L+1)(B-M+1)(C-N+1)$ enhanced matrix $\mathbf{D}_{e}$ from the noisy data according to (15).

Step 2. Compute the singular values and the left singular vectors $\mathbf{U}_{s}$ of $\mathbf{D}_{e}$. Estimate the number of the sources from the singular values.

Step 3. Estimate the poles $x_{i}, y_{i}$, and $z_{i}$ from $\mathbf{U}_{s}$ and pair the poles as illustrated in [9].

Step 4. Estimate the 2D DOA of coherently distributed source from the poles by using (11) and (12).

The MP algorithm for 2D DOA estimation only used the phase information of the signal. It can be inferred that the angular spread can be got from the module information of the signal with some prior information of the angular weighting function. From literature [9], the wavelength can be got from the estimated poles. However, for simplicity, in this paper, the 2D DOA estimation problem for coherently distributed sources is focused.

\section{CRAMER-RAO BOUND}

The Cramer-Rao bound (CRB) for the point source could be seen in [9]; the CRB for coherently distributed source is derived as follows.

Consider the sampled values of the noise contaminated signal $\tilde{v}$. Assume that the noise is complex Gaussian, the probability density function of $\tilde{v}$ is

$$
P(\tilde{v} / \varphi)=\frac{1}{(2 \pi \kappa)^{A B C}} e^{\left((-1 / \kappa)\|\tilde{v}-v\|^{2}\right)},
$$

where $\|\cdot\|$ denotes the 2-norm, $\kappa$ is the variance of the noise, and $\varphi$ is the $I \times 1$ column vector of the unknown parameters defined as follows:

$$
\begin{aligned}
\boldsymbol{\varphi} & =\left[\begin{array}{llll}
\boldsymbol{\varphi}_{1} & \boldsymbol{\varphi}_{2} & \cdots & \boldsymbol{\varphi}_{I}
\end{array}\right]^{T}, \\
\boldsymbol{\varphi}_{i} & =\left[\begin{array}{lllllll}
M_{i} & \gamma_{i} & \lambda_{i} & \theta_{i} & \phi_{i} & \sigma_{\theta_{i}} & \sigma_{\phi_{i}}
\end{array}\right]^{T} .
\end{aligned}
$$

The element of the $7 I \times 7 I$ Fisher information matrix $\mathbf{F}$ is defined by

$$
\mathbf{F}_{i j}=-E\left\{\frac{\partial^{2}}{\partial \varphi_{i} \partial \varphi_{j}} \log (p(\tilde{v} / \varphi))\right\},
$$




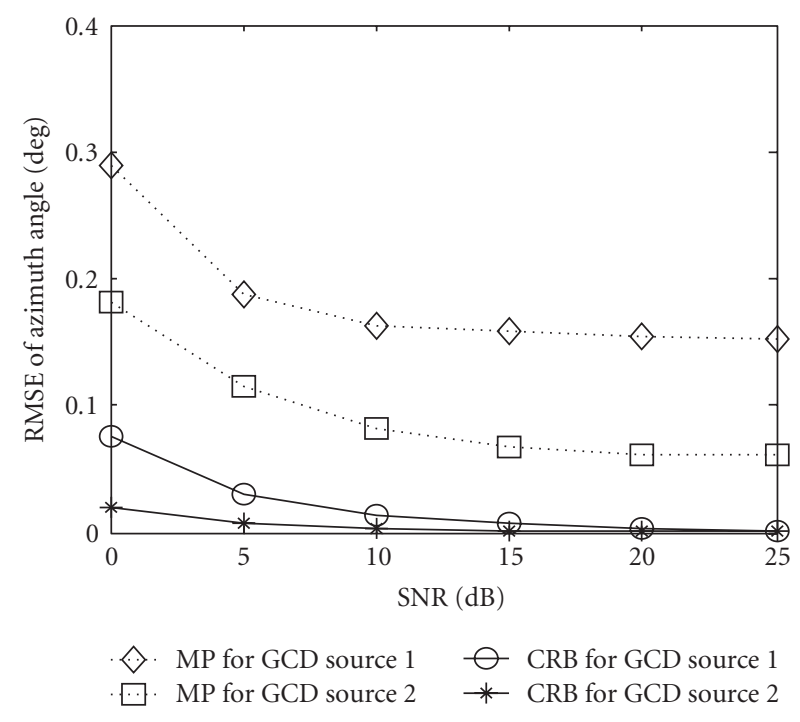

Figure 2: RMSE of azimuth angle for GCD sources.

where $\mathbf{F}_{i j}$ is a $7 \times 7,(i, j)$ th block matrix of $\mathbf{F} . E\{\cdot\}$ is the expectation operator, $\partial / \partial \varphi_{i}$ is the partial derivative with respect to the $i$ th element $\varphi_{i}$ of $\varphi$, and $\log$ is the natural logarithm. Using (18) in (20), we have

$$
\mathbf{F}_{i j}=\frac{1}{\kappa} 2 \operatorname{Re}\left(\frac{\partial v^{H}}{\partial \varphi_{i}} \frac{\partial v}{\partial \varphi_{j}}\right),
$$

where $\operatorname{Re}(\cdot)$ denotes the real part.

By using the Fisher information matrix, the Cramer-Rao bound $(\mathrm{CRB})$ is defined as

$$
\operatorname{var}\left(\boldsymbol{\varphi}_{i}\right) \geq\left[\mathbf{F}^{-1}(\boldsymbol{\varphi})\right]_{i i}
$$

So the variance of the unbiased estimate of the $i$ th parameter is the $i$ th diagonal element of the inverse of the Fisher information matrix. Thus, we can compare the RMSE of the MP algorithm with $\sqrt{\operatorname{var}\left(\varphi_{i}\right)}$ to measure the goodness of the estimator.

\section{NUMERICAL RESULTS}

In this section, we provide numerical illustrations of the performance of the proposed algorithm. We assume all of the signals are equipower and have the same frequency. The numbers of the array elements in $x$-direction, $y$-direction, and $z$-direction are all 10, the distance between adjacent sensors is $\lambda / 2$. It is assumed that all the signals impinging on the array with amplitudes $M_{i}=1$ and phases $\gamma_{i}=0$. The results are based on 500 Monte Carlo simulations.

In the first example, we illustrate the performance of MP algorithm for two GCD source one with $\mu_{1}=(35,3.5,40,4)$ and another one with $\mu_{2}=(25,2.5,20,2)$. The pencil parameters are all 4 . We compare with the CRB for $2 \mathrm{D}$ DOA estimation of Gaussian shaped coherently distributed source. Figures 2 and 3 show that the RMSE of the estimators approaches the CRB when SNR varies from $0 \mathrm{~dB}$ to $25 \mathrm{~dB}$.

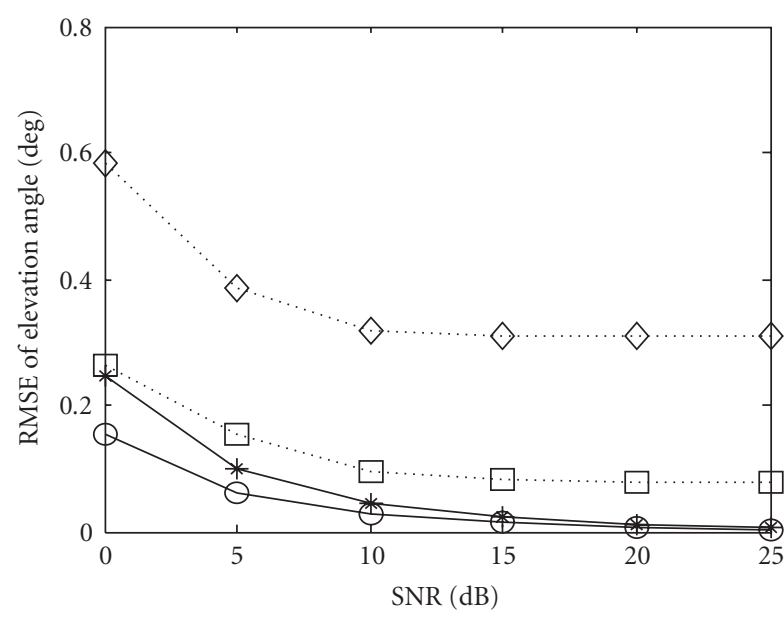

$\odot$ MP for GCD source $1 \bigcirc$ CRB for GCD source 1 . MP for GCD source $2 \rightarrow$ CRB for GCD source 2

Figure 3: RMSE of elevation angle for GCD sources.

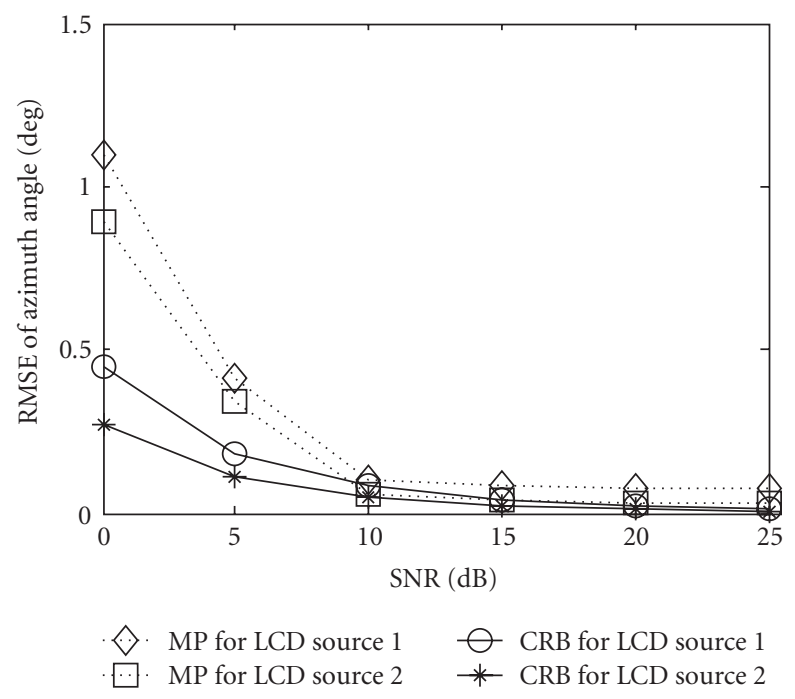

Figure 4: RMSE of azimuth angle for LCD sources.

The RMS errors for the two GCD sources using MP are all smaller than 1 degree when the SNR at $0 \mathrm{~dB}$.

In the second example, we illustrate the performance of MP algorithm for two LCD source, one with $\mu_{1}=$ $(35,3.5,40,4)$ and another one with $\mu_{2}=(25,2.5,20,2)$. The pencil parameters are all 4 . Figures 4 and 5 show that the RMSE of the estimators approaches the CRB when SNR varies from $0 \mathrm{~dB}$ to $25 \mathrm{~dB}$. The RMS errors for the two LCD source using MP are smaller than 1 degree when the SNR at $0 \mathrm{~dB}$.

In the third example, we first illustrate the performance of MP algorithm when $\theta=35^{\circ}, \phi=40^{\circ}, \sigma_{\phi}=4^{\circ}$, the angular spread $\sigma_{\theta}$ varies, the pencil parameters are all 4 , and SNR is $10 \mathrm{~dB}$ : it is observed that the variation of RMSE of azimuth angle is rather small even when $\sigma_{\theta}$ (tdelta in Figure 6) increases. We then illustrate the performance of MP 


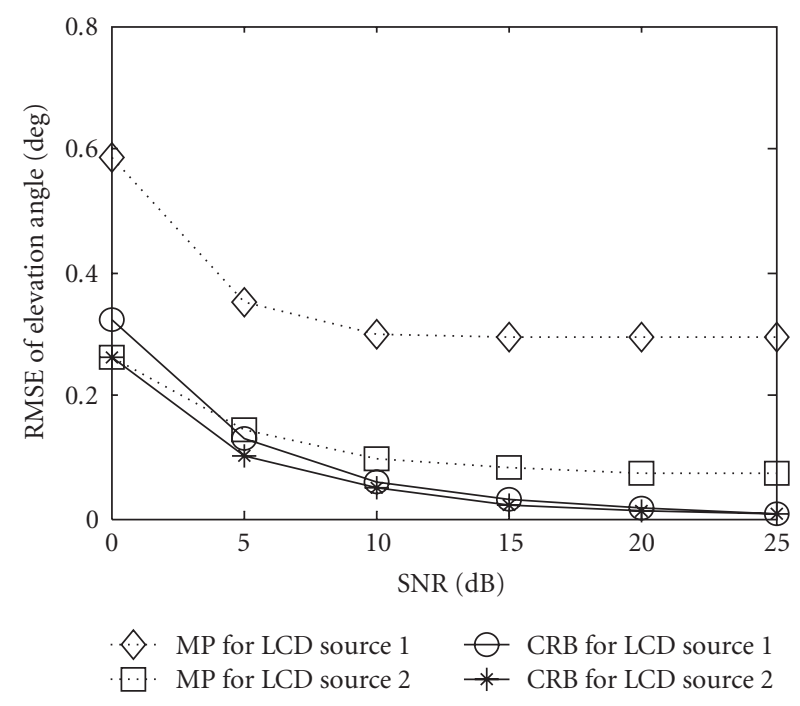

FIGURE 5: RMSE of elevation angle for LCD sources.



FIgURE 6: RMSE of azimuth angle versus $\sigma_{\theta}$.

algorithm when $\theta=35^{\circ}, \phi=40^{\circ}, \sigma_{\theta}=4^{\circ}$, the angular spread $\sigma_{\phi}$ varies, also the pencil parameters are all 4 , and SNR is $10 \mathrm{~dB}$ : it is observed that the variation of RMSE of elevation angle is also rather small even when $\sigma_{\phi}$ (fdelta in Figure 7) increases.

Clearly, the MP algorithm provides good estimation accuracy for estimating the nominal azimuth and elevation DOA of coherently distributed source. Note that because the angular information of coherently distributed source is separated from angular spread information, the estimation of the 2D DOA does not need the information of the shape of angular weighting function.

\section{CONCLUSIONS}

In this study, the coherently distributed source with $3 \mathrm{D}$ data cube is constructed using the Taylor approximation, whereas the angular and the angular spread information is

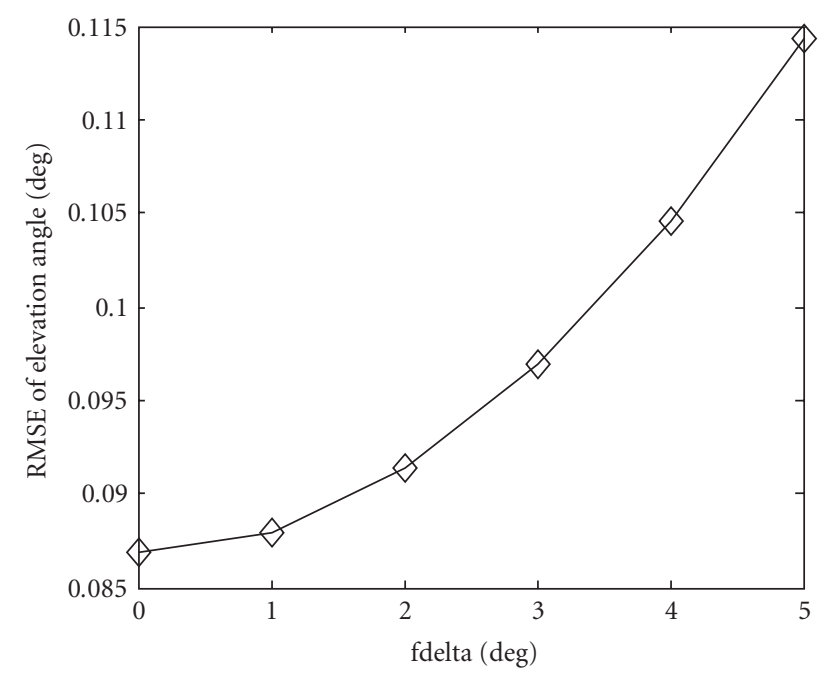

FIgURE 7: RMSE of elevation angle versus $\sigma_{\phi}$.

separated from the signal pattern. The matrix pencil method is extended to the estimation of $2 \mathrm{D} \mathrm{DOA}$ for coherently distributed sources without any search. 3D data matrix is constructed to estimate poles of 3D plane, the azimuth and elevation of each signal could be obtained from the poles. This method could deal with differently shaped small angular spread coherently distributed sources without the prior information of the shape of the angular weighting function. Computer simulation validated the efficiency of the method. The estimation performance of different shaped coherently distributed source is studied. The RMS errors of the estimator have been compared with the CRB to observe the goodness of the method at low SNR.

\section{APPENDIX}

\section{APPROXIMATION TO THE STEERING VECTOR FOR SMALL ANGULAR SPREADS}

From (2), we have

$$
\begin{aligned}
{[\mathbf{b}(\mu)]=} & {\left[\mathbf{b}\left(\theta, \sigma_{\theta}, \phi, \sigma_{\phi}\right)\right] } \\
= & \iint[\mathbf{a}(\vartheta, \varphi)] \rho(\vartheta, \varphi ; \mu) d \vartheta d \varphi \\
= & \iint e^{j 2 \pi(\Delta x \cos \theta \cos \phi a+\Delta y \sin \theta \cos \phi b+\Delta z \sin \phi c) / \lambda} \\
& \quad \times \rho(\widetilde{\vartheta}+\theta, \tilde{\varphi}+\phi ; \mu) d \widetilde{\vartheta} d \widetilde{\varphi},
\end{aligned}
$$

where $\mu=\left(\theta, \sigma_{\theta}, \phi, \sigma_{\phi}\right)$ characterizes the complex source together with the angular weighting function $\rho(\vartheta, \varphi ; \mu)$ which shows the angular spreading of the source, for instance, the Gaussian shaped angular weighting function can be expressed as

$$
\rho(\vartheta, \varphi ; \mu)=\frac{1}{2 \pi \sigma_{\theta} \sigma_{\phi}} e^{-1 / 2\left((9-\theta)^{2} / \sigma_{\theta}^{2}+(\varphi-\phi)^{2} / \sigma_{\phi}^{2}\right)} .
$$

For small values of variables $\tilde{\vartheta}=\vartheta-\theta$ and $\tilde{\varphi}=\varphi-\phi$, the functions $\sin \tilde{\vartheta}, \cos \tilde{\vartheta}, \sin \tilde{\varphi}$, and $\cos \tilde{\varphi}$ can be approximated 
by the first terms in the Taylor series expansions. Using the trigonometric identity $\cos (\alpha+\beta)=\cos \alpha \cos \beta-\sin \alpha \sin \beta$ and $\sin (\alpha+\beta)=\sin \alpha \cdot \cos \beta+\cos \alpha \sin \beta$, we have

$$
\begin{aligned}
& e^{j 2 \pi(\Delta x \cos (\theta+\widetilde{\vartheta}) \cos (\phi+\widetilde{\varphi}) a+\Delta y \sin (\theta+\widetilde{\vartheta}) \cos (\phi+\tilde{\varphi}) b+\Delta z \sin (\phi+\tilde{\varphi}) c) / \lambda} \\
&=e^{j 2 \pi(\Delta x \cos (\theta+\tilde{\vartheta}) \cos (\phi+\widetilde{\varphi}) a) / \lambda} \\
& \quad \times e^{j 2 \pi(\Delta y \sin (\theta+\widetilde{\vartheta}) \cos (\phi+\widetilde{\varphi}) b) / \lambda} \times e^{j 2 \pi(\Delta z \sin (\phi+\widetilde{\varphi}) c) / \lambda} \\
& \approx e^{j 2 \pi(\Delta x(\cos \theta-\tilde{\vartheta} \sin \theta)(\cos \phi-\tilde{\varphi} \sin \phi) a) / \lambda} \\
& \times e^{j 2 \pi(\Delta y(\sin \theta+\widetilde{\vartheta} \cos \theta)(\cos \phi-\tilde{\varphi} \sin \phi) b) / \lambda} \\
& \times e^{j 2 \pi(\Delta z(\sin \phi+\tilde{\varphi} \cos \phi) c) / \lambda} \\
& \simeq e^{j 2 \pi(\Delta x \cos (\theta) \cos (\phi) a+\Delta y \sin (\theta) \cos (\phi) b+\Delta z \sin (\phi) c) / \lambda} \\
& \times e^{j 2 \pi \widetilde{\vartheta}(-\Delta x \sin \theta \cos \phi a+\Delta y \cos \theta \cos \phi b) / \lambda} \\
& \times e^{j 2 \pi \widetilde{\varphi}(-\Delta x \cos \theta \sin \phi a-\Delta y \sin \theta \sin \phi b+\Delta z \cos \phi c) / \lambda},
\end{aligned}
$$

where we assume that $\tilde{\vartheta} \tilde{\varphi} \approx 0$ and consequently $e^{j 2 \pi \tilde{\vartheta} \tilde{\varphi} \sin \theta \sin \phi / \lambda} \simeq 1, e^{j 2 \pi \tilde{\vartheta} \tilde{\varphi} \cos \theta \sin \phi / \lambda} \simeq 1$. Thus, we can rewrite (A.1) as

$$
\mathbf{b}\left(\theta, \sigma_{\theta}, \phi, \sigma_{\phi}\right) \approx \mathbf{a}(\theta, \phi) \odot \mathbf{g}\left(\theta, \sigma_{\theta}, \phi, \sigma_{\phi}\right),
$$

or

$$
\left[\mathbf{b}\left(\theta, \sigma_{\theta}, \phi, \sigma_{\phi}\right)\right]_{(a, b, c)} \approx[\mathbf{a}(\theta, \phi)]_{(a, b, c)}\left[\mathbf{g}\left(\theta, \sigma_{\theta}, \phi, \sigma_{\phi}\right)\right]_{(a, b, c)},
$$

where

$$
\begin{aligned}
& {\left[\mathbf{g}\left(\theta, \sigma_{\theta}, \phi, \sigma_{\phi}\right)\right]_{(a, b, c)}} \\
& =\iint e^{j 2 \pi \widetilde{\vartheta}(-\Delta x \sin \theta \cos \phi a+\Delta y \cos \theta \cos \phi b) / \lambda} \\
& \quad \times e^{j 2 \pi \widetilde{\varphi}(-\Delta x \cos \theta \sin \phi a-\Delta y \sin \theta \sin \phi b+\Delta z \cos \phi c) / \lambda} \\
& \quad \times \rho(\widetilde{\vartheta}+\theta, \tilde{\varphi}+\phi ; \mu) d \widetilde{\vartheta} d \tilde{\varphi}
\end{aligned}
$$
have

For Gaussian shaped angular weighting function, we

$$
\begin{aligned}
& {\left[\mathbf{g}\left(\theta, \sigma_{\theta}, \phi, \sigma_{\phi}\right)\right]_{(a, b, c)}} \\
& =\frac{1}{2 \pi \sigma_{\theta} \sigma_{\phi}} \int e^{j 2 \pi \widetilde{\vartheta}(-\Delta x \sin \theta \cos \phi a+\Delta y \cos \theta \cos \phi b) / \lambda} e^{-\left(\widetilde{\vartheta}^{2} / 2 \sigma_{\theta}^{2}\right)} d \widetilde{\vartheta} \\
& \quad \times \int e^{j 2 \pi \widetilde{\varphi}(-\Delta x \cos \theta \sin \phi a-\Delta y \sin \theta \sin \phi b+\Delta z \cos \phi c) / \lambda} e^{-\left(\widetilde{\varphi}^{2} / 2 \sigma_{\phi}^{2}\right)} d \widetilde{\varphi} \\
& =e^{-2 \pi^{2} \sigma_{\theta}^{2}(-\Delta x \sin \theta \cos \phi a+\Delta y \cos \theta \cos \phi b)^{2} / \lambda^{2}} \\
& \quad \times e^{-2 \pi^{2} \sigma_{\phi}^{2}(-\Delta x \cos \theta \sin \phi a-\Delta y \sin \theta \sin \phi b+\Delta z \cos \phi c)^{2} / \lambda^{2}},
\end{aligned}
$$

where the integral formula $\int_{-\infty}^{\infty} e^{-q^{2} x^{2}} e^{j p(x+\lambda)} d x=\sqrt{\pi} e^{j p \lambda}$. $e^{-\left(p^{2} / 4 q^{2}\right)} / q$ is used.

Similarly, when the angular weighting function is Laplacian shaped:

$$
\rho(\tilde{\vartheta}, \tilde{\varphi} ; \mu)=\frac{1}{2 \sigma_{\theta} \sigma_{\phi}} e^{-\left(\sqrt{2}|\widetilde{\vartheta}-\theta| / \sigma_{\theta}+\sqrt{2}|\tilde{\varphi}-\phi| / \sigma_{\phi}\right)},
$$

we have

$$
\begin{aligned}
& {\left[b\left(\theta, \sigma_{\theta}, \phi, \sigma_{\phi}\right)\right]_{(a, b, c)}} \\
& \approx[\mathbf{a}(\theta, \phi)]_{(a, b, c)}\left[\mathbf{g}\left(\theta, \sigma_{\theta}, \phi, \sigma_{\phi}\right)\right]_{(a, b, c)} \\
& \simeq[\mathbf{a}(\theta, \phi)]_{(a, b, c)} \\
& \quad \times\left(\frac{1}{\sqrt{2} \sigma_{\theta}} \int e^{j 2 \pi \tilde{\vartheta}(-\Delta x \sin \theta \cos \phi a+\Delta y \cos \theta \cos \phi b) / \lambda} e^{-\left(\sqrt{2}|\tilde{\vartheta}| / \sigma_{\theta}\right)} d \widetilde{\vartheta}\right) \\
& \quad \times\left(\frac{1}{\sqrt{2} \sigma_{\phi}} \int e^{j 2 \pi \tilde{\varphi}(-\Delta x \cos \theta \sin \phi a-\Delta y \sin \theta \sin \phi b+\Delta z \cos \phi c) / \lambda}\right. \\
& \left.\quad \times e^{-\left(\sqrt{2}|\tilde{\varphi}| / \sigma_{\phi}\right)} d \tilde{\varphi}\right) \\
& \simeq \quad[\mathbf{a}(\theta, \phi)]_{(a, b, c)} \\
& \quad \times 1 /\left(1+2\left(\pi \sigma_{\theta}(-\Delta x \sin \theta \cos \phi a+\Delta y \cos \theta \cos \phi b) / \lambda\right)^{2}\right) \\
& \quad \times 1 /\left(1+2\left(\pi \sigma_{\phi}(-\Delta x \cos \theta \sin \phi a-\Delta y \sin \theta \sin \phi b\right.\right. \\
& \left.\quad+\Delta z \cos \phi c) / \lambda)^{2}\right)
\end{aligned}
$$

using $\int_{0}^{\infty} e^{-p x} \cos (v x+\varepsilon) d x=(p \cos \varepsilon-v \sin \varepsilon) /\left(p^{2}+v^{2}\right)$, $p>0$.

\section{REFERENCES}

[1] P. Zetterberg, Mobile cellular communications with base station antenna arrays: spectrum efficiency, algorithms and propagation models, Ph.D. dissertation, Signals, Sensors, Systems Department, Royal Institute of Technology, Stockholm, Sweden, 1997.

[2] S. Valaee, B. Champagne, and P. Kabal, "Parametric localization of distributed sources," IEEE Transactions on Signal Processing, vol. 43, no. 9, pp. 2144-2153, 1995.

[3] S. Shahbazpanahi, S. Valaee, and M. H. Bastani, "Distributed source localization using ESPRIT algorithm," IEEE Transactions on Signal Processing, vol. 49, no. 10, pp. 2169-2178, 2001.

[4] J. Lee, I. Song, H. Kwon, and S. R. Lee, "Low-complexity estimation of 2D DOA for coherently distributed sources," Signal Processing, vol. 83, no. 8, pp. 1789-1802, 2003.

[5] G. Y. Zhang and T. Bin, "Estimation of 2D-DOAs and angular spreads for coherently distributed sources using cumulants," in Proceedings of the 8th IEEE Workshop on Signal Processing Advances in Wireless Communications (SPAWC '07), pp. 1-5, Helsinki, Finland, June 2007.

[6] A. Zoubir and Y. Wang, "Efficient DSPE algorithm for estimating the angular parameters of coherently distributed sources," Signal Processing, vol. 88, no. 4, pp. 1071-1078, 2008.

[7] Y. B. Hua, "Estimating two-dimensional frequencies by matrix enhancement and matrix pencil," IEEE Transactions on Signal Processing, vol. 40, no. 9, pp. 2267-2280, 1992.

[8] Y. B. Hua and T. K. Sarkar, "Matrix pencil method for estimating parameters of exponentially damped/undamped sinusoids in noise," IEEE Transactions on Acoustics, Speech, and Signal Processing, vol. 38, no. 5, pp. 814-824, 1990.

[9] N. Yilmazer, R. Fernandez-Recio, and T. K. Sarkar, "Matrix pencil method for simultaneously estimating azimuth and elevation angles of arrival along with the frequency of the 
incoming signals," Digital Signal Processing, vol. 16, no. 6, pp. 796-816, 2006.

[10] N. Yilmazer, J. Koh, and T. K. Sarkar, "Utilization of a unitary transform for efficient computation in the matrix pencil method to find the direction of arrival," IEEE Transactions on Antennas and Propagation, vol. 54, no. 1, pp. 175-181, 2006. 